\title{
On the Embedding Phase of the Hopcroft and Tarjan Planarity Testing Algorithm
}

\author{
Kurt Mehlhorn and Petra Mutzel *i
}

\begin{abstract}
We give a detailed description of the embedding phase of the Hopcroft and Tarjan planarity testing algorithm. The embedding phase runs in linear time. An implementation based on this paper can be found in [MMN93].
\end{abstract}

An undirected graph $G=(V, E)$ is called planar if it can be mapped into the plane without edge crossings, i.e., the vertices of $G$ are mapped into distinct points in the plane and the edges of $G$ are mapped into disjoint Jordan curves connecting their endpoints. Such a mapping is called a planar geometric embedding. Two geometric planar embeddings are called equivalent if there is an homeomorphism of the plane transforming one into the other. An equivalence class of geometric planar embeddings is called a planar topological embedding or simply planar embedding.

A planar embedding of a planar graph induces a cyclic ordering on the edges incident to any fixed vertex, namely the clockwise ordering of the edges around their common endpoint. A graph $G$ together with a cyclic ordering on the edges incident to each vertex is called a combinatorial embedding, it is called a planar combinatorial embedding if it is induced by some planar embedding. Different planar embeddings can give rise to the same combinatorial embedding. However, a planar combinatorial embedding of a connected graph uniquely determines its topological embedding on the sphere. In the plane, it determines the topological embedding up to selection of the outer face. Recall that an embedding into the plane can be obtained from an embedding on the sphere by polar projection. The pole can be put into any face. There are linear time algorithms [dFPP91, Sch90] to convert a planar combinatorial embedding into a geometric embedding, e.g., the algorithm by Schnyder puts the vertices of an $n$ node graph onto an $(n-2) \times(n-2)$ grid and realizes the edges by straight-line segments.

Hopcroft and Tarjan [HT74] gave an algorithm that tests the planarity of an undirected graph in linear time. Alternative linear time algorithms were developed by Lempel, Even, and Cederbaum [LEC67, ET76], Booth and Lueker [BL76], and Fraysseix and Rosenstiehl [FR82]. Chiba, Nishizeki, Abe and Ozawa [CNAO85] have shown how to extend the algorithm of Booth and Lueker so as to also construct a planar combinatorial embedding. Hopcroft and Tarjan also stated but gave no details that their planarity testing algorithm can be extended to also construct a planar combinatorial embedding. The textbook of the first author [Meh84, vol. 2, page 112] attempts to give more details (in less than one page) but the presentation is incorrect. We conclude that there is no published correct presentation of the embedding phase of the Hopcroft and Tarjan algorithm.

\footnotetext{
${ }^{*}$ Max-Planck-Institut für Informatik, Im Stadtwald, 66123 Saarbrücken, Germany

† Revised version, 09.03.1995
} 


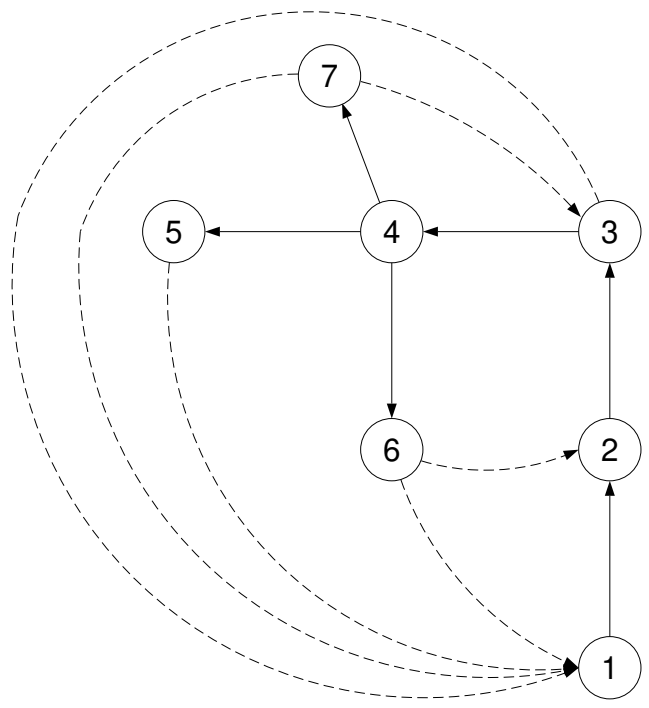

Figure 1: A DFS-tree of a planar graph. Tree edges are shown solid and back edges are shown dashed.

In this note we give a complete description of the embedding phase. An alternative presentation can be found in [Mut92]. Our embedding algorithm has the same recursive structure as the testing algorithm of Hopcroft and Tarjan and also runs in linear time. An implementation based on this note is described in [MMN93] and is distributed with the LEDA platform of combinatorial and geometric computing [Näh95, MN95] (anonymous ftp mpi-sb.mpg.de, directory pub/LEDA).

The testing phase of the Hopcroft and Tarjan algorithm is discussed in detail in [Meh84, vol. 2, pages $96-111$ ]. We summarize that discussion. The graph $G$ is assumed to be biconnected. We also fix a particular DFS-tree of $G$ and identify the vertices of $G$ with their DFS-numbers. We direct all tree edges from lower to higher DFS-number and all non-tree edges from higher to lower DFS-number. Non-tree edges are called back edges. Figure 1 shows an example. We use $T$ and $B$ to denote the set of tree edges and back edges respectively.

We associate a segment $S(e)$ and a cycle $C(e)$ with every edge $e=(x, y)$ of $G$. If $e$ is a back edge then $C(e)$ and $S(e)$ consist of the tree path from $y$ to $x$ and the edge $e$. If $e$ is a tree edge then let $V(e)$ be the set of tree successors of $y$ (including $y$ itself) and let $w_{0}$ be the lowest numbered endpoint of any back edge starting in $V(e)$. The cycle $C(e)$ consists of a tree path from the vertex $w_{0}$ to a vertex $w \in V(e)$ with $\left(w, w_{0}\right) \in B$ and the back edge $\left(w, w_{0}\right)$ and the segment $S(e)$ consists of $C(e)$, the subgraph induced by $V(e)$, and all back edges starting in a node in $V(e)$. Note that the segment $S(e)$ is uniquely defined but that there may be several choices for the cycle $C(e)$. We will later fix one particular choice for $C(e)$. We divide the tree path underlying the cycle $C(e)$ into two parts, its stem and its spine. The stem consists of the part ending in $x$. The spine is empty if $e$ is a back edge and it is the part starting in $y$ if $e$ is a tree edge.

In our example, the cycle $C((3,4))$ may consist of the tree path from 1 to 5 followed by the back edge $(5,1)$. The stem is the tree path from 1 to 3 and the spine is the tree path from 4 to 5 . The cycle $C((1,2))$ may consist of the tree path from 1 to 3 and the back edge $(3,1)$. 

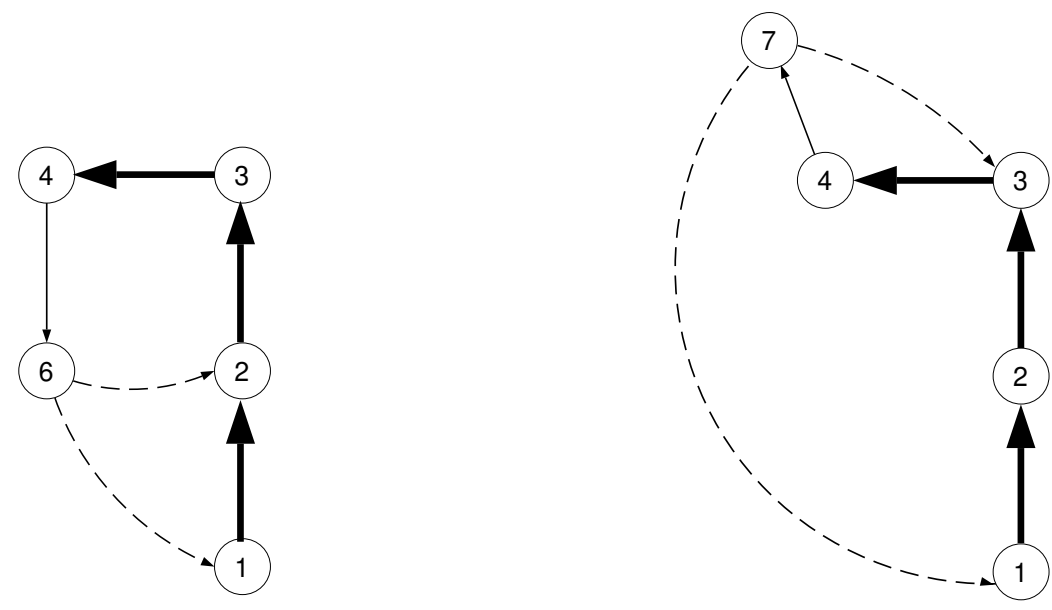

Figure 2: Embeddings of $S((4,6))$ and $S((4,7))$ induced by the embedding of $G$. Stems are shown in bold. The embedding of $S((4,6))$ is canonical and the embedding of $S((4,7))$ is reversed canonical.

Its stem is the node 1 and its spine is the tree path from 2 to 3 . The segment $S((1,2))$ is the entire graph $G$ and the segment $S((3,4))$ is the graph $G$ minus the edge $(3,1)$.

A segment $S(e)$ is called strongly planar if there is a planar embedding of $S(e)$ and some face in that embedding such that the entire stem of the cycle $C(e)$ is contained in the border of the face. An embedding with such a property is called a strongly planar embedding of $S(e)$. If a segment $S(e)$ has a strongly planar embedding then it also has one where the stem of $C(e)$ borders the outer face. When we talk about a strongly planar embedding we assume from now on that the stem of $C(e)$ borders the outer face. Let $w_{0}, w_{1}, \ldots, w_{r}$ with $e=\left(w_{r}, y\right)$ be the stem of $C(e)$. A strongly planar embedding of $S(e)$ is called canonical (reversed canonical) if for all $i, 0<i<r$, the edge $\left(w_{i}, w_{i+1}\right)$ immediately precedes (follows) the edge $\left(w_{i}, w_{i-1}\right)$ in the clockwise ordering of edges incident to $w_{i}$.

Figure 2 shows the embeddings of $S((4,6))$ and $S((4,7))$ induced by the embedding of $G$ shown in Figure 1.

Since $G$ is assumed to be biconnected there is exactly one tree edge out of vertex 1 , namely the edge $(1,2)$. Moreover $G=S((1,2))$ and $G$ is planar iff $S((1,2))$ is strongly planar.

Let $e_{0}$ be any edge and let $C=C\left(e_{0}\right)$ be the cycle associated with $e_{0}$. An edge $e=(x, y)$ is said to emanate from $C$ if $x$ lies on the spine of $C$ but $e$ does not belong to $C$. We will also say that the segment $S(e)$ emanates from $C$. If $e_{1}, \ldots, e_{m}$ are the edges emanating from $C$ then $S\left(e_{0}\right)=C+S\left(e_{1}\right)+\ldots+S\left(e_{m}\right)$, i.e., $S\left(e_{0}\right)$ is the union of the cycle $C$ and the segments $S\left(e_{1}\right), \ldots, S\left(e_{m}\right)$.

We need some more concepts. As above, let $C=C\left(e_{0}\right)$ and let $e=(x, y)$ emanate from $C$. The set $A(e)$ of attachments of segment $S(e)$ to cycle $C$ is defined to be the set $\{x, y\}$ if $e$ is a back edge and the set $\{x\} \cup\{z ;(w, z)$ is a back edge, $w \in V(e)$ and $z \notin V(e)\}$ if $e$ is a tree edge. Two segments $S(e)$ and $S\left(e^{\prime}\right)$ where $e$ and $e^{\prime}$ emanate from $C$ are said to interlace if either there are nodes $x<y<z<u$ on cycle $C$ such that $x, z \in A(e)$ and $y, u \in A\left(e^{\prime}\right)$ or $A(e)$ and $A\left(e^{\prime}\right)$ have three points in common.

The interlacing graph $I G(C)$ with respect to cycle $C=C\left(e_{0}\right)$ is defined as follows. The nodes of $I G(C)$ are the segments $S(e)$ where $e$ emanates from $C$. Also, $S(e)$ and $S\left(e^{\prime}\right)$ are 
connected by an edge iff $S(e)$ and $S\left(e^{\prime}\right)$ interlace. The segment $S\left(e_{0}\right)$ is strongly planar iff the following conditions hold. Firstly, $S(e)$ is strongly planar for every $e$ emanating from $C$. Secondly, there is a partition $\{L, R\}$ of the segments emanating from $C$ such that no two segments in $L$ or $R$ interlace and such that $A(e) \cap\left\{w_{1}, \ldots w_{r-1}\right\}=\emptyset$ for any segment $S(e) \in R$, where $w_{0}, w_{1}, \ldots w_{r-1}, w_{r}$ is the stem of cycle $C$.

For reasons of efficiency, it is useful to order the adjacency list of any vertex $v$ as follows: edge $(v, w)$ is before edge $\left(v, w^{\prime}\right)$ if $\min A((v, w))<\min A\left(\left(v, w^{\prime}\right)\right)$ or if $\min A((v, w))=$ $\min A\left(\left(v, w^{\prime}\right)\right), A((v, w))$ has cardinality two, and $A\left(\left(v, w^{\prime}\right)\right)$ has cardinality three or more. In all other cases the order is irrelevant. We assume from now on that the cycle $C(e)$ for a tree edge $e=(x, y)$ is defined in the following way. Starting in $y$ we construct a path by always taking the first edge out of each node until a back edge is encountered. The path constructed this way is the spine of the cycle $C(e)$.

The discussion above suggests a procedure stronglyplanar $\left(e_{0}\right), \mathrm{cf}$. [Meh84, vol. 2, page 109] that given an edge $e_{0}$ decides the strong planarity of the segment $S\left(e_{0}\right)$. It first constructs the cycle $C=C\left(e_{0}\right)$, then recursively tests the strong planarity of all segments $S(e)$, where $e$ emanates from $C$, and finally tests, whether there is an appropriate bipartition of the vertex set of the interlacing graph. The recursive calls are made in the following order. If $w_{r+1}, \ldots, w_{k}$ is the spine of the cycle $C$ then the segments $S\left(\left(w_{k},\right)\right)$ are tested first, the segments $S\left(\left(w_{k-1},\right)\right)$ are tested next,... . For each fixed $i$ the segments $S\left(\left(w_{i},\right)\right)$ are tested in the order in which the edges $\left(w_{i},\right)$ appear on the adjacency list of $w_{i}$. The call stronglyplanar $((1,2))$ tests the strong planarity of segment $S((1,2))$ and hence the planarity of $G$.

As shown in [Meh84, vol. 2, page 112] procedure stronglyplanar can also be used to compute a labelling $\alpha$ of the edges of $G$ by $L$ and $R$ such that:

- an edge $e$ is labelled iff stronglyplanar( $e$ ) is called

- edge $(1,2)$ is labelled $L$

- if $e_{0}$ is a labelled edge and $e_{1}, \ldots, e_{m}$ are the edges emanating from $C=C\left(e_{0}\right)$ then $\alpha$ induces the appropriate bipartition of the interlacing graph, i.e., if $\alpha\left(e_{i}\right)=\alpha\left(e_{j}\right)$ then $S\left(e_{i}\right)$ and $S\left(e_{j}\right)$ do not interlace and if $\alpha\left(e_{j}\right)=R$ then $A\left(e_{j}\right) \cap\left\{w_{1}, \ldots, w_{r-1}\right\}=\emptyset$ where $w_{0}, \ldots, w_{r}$ is the stem of $C$.

The correctness proof of procedure stronglyplanar demonstrates how a strongly planar embedding of $S\left(e_{0}\right)$ can be obtained from strongly planar embeddings of the $S\left(e_{i}\right)$ 's:

To construct a canonical embedding of $S\left(e_{0}\right)$ draw the path $w_{0}, \ldots, w_{k}$ (consisting of stem $w_{0}, \ldots, w_{r}$, edge $e_{0}=\left(w_{r}, w_{r+1}\right)$ and spine $\left(w_{r+1}, \ldots, w_{k}\right)$ as a vertical upwards directed path, add edge $\left(w_{k}, w_{0}\right)$, and then for $i, 1 \leq i \leq m$, and $\alpha\left(e_{i}\right)=L$ extend the embedding of $C+S\left(e_{1}\right)+\ldots S\left(e_{i-1}\right)$ by glueing a canonical embedding of $S\left(e_{i}\right)$ onto the left side of the vertical path, and for $i, 1 \leq i \leq m$, and $\alpha\left(e_{i}\right)=R$ extend the embedding of $C+S\left(e_{1}\right)+\ldots+S\left(e_{i-1}\right)$ by glueing a reversed canonical embedding of $S\left(e_{i}\right)$ onto the right side of the vertical path. Similarly, if the goal is to construct a reversed canonical embedding of $S\left(e_{0}\right)$ then, if $\alpha\left(e_{i}\right)=L$, a reversed canonical embedding of $S\left(e_{i}\right)$ is glued onto the right side of the vertical path, and if $\alpha\left(e_{i}\right)=R$, then a canonical embedding of $S\left(e_{i}\right)$ is glued onto the left side of the vertical path. This completes the review of [Meh84].

We can now give the algorithmic details. We first use procedure stronglyplanar to compute the mapping $\alpha$. We then use a procedure embedding to actually compute an embedding. The procedure embedding takes two parameters: a tree edge $e_{0}$ and a flag $t \in\{L, R\}$. A call embedding $\left(e_{0}, L\right)$ computes a canonical embedding of $S\left(e_{0}\right)$ and a call embedding $\left(e_{0}, R\right)$ 
(0) procedure embedding $\left(e_{0}\right.$ : edge, $\left.t:\{L, R\}\right)$

(* computes an embedding of $S\left(e_{0}\right), e_{0}=(x, y)$, as described in the text;

it returns the lists $T$ and $A$ defined in the text $*$ )

(1) find the spine of segment $S\left(e_{0}\right)$ by starting in node $y$ and always

taking the first edge of every adjacency list until a back edge is encountered. This back edge leads to node $w_{0}$.

(* Let $w_{0}, \ldots, w_{r}$ be the tree path from $w_{0}$ to $x=w_{r}$ and

let $w_{r+1}=y, \ldots, w_{k}$ be the spine constructed above. $*$ )

(2) $A L \leftarrow A R \leftarrow$ empty list of edges;

$T \leftarrow\left(w_{k}, w_{0}\right) ;$

(3) for $j$ from $k$ downto $r+1$

(4) do for all edges $e^{\prime}$ (except the first) emanating from $w_{j}$

(5) do if $e^{\prime}$ is a tree edge

(6) then $\left(T^{\prime}, A^{\prime}\right) \leftarrow$ embedding $\left(e^{\prime}, t \oplus \alpha\left(e^{\prime}\right)\right)$

(7) else $T^{\prime} \leftarrow\left(e^{\prime}\right), A^{\prime} \leftarrow$ (reversal of $\left.e^{\prime}\right)$

(8) fi

(9) if $t=\alpha\left(e^{\prime}\right)$

(10) then $T \leftarrow T^{\prime}$ conc $T ; A L \leftarrow A L$ conc $A^{\prime}$

(11) else $T \leftarrow T$ conc $T^{\prime} ; A R \leftarrow A^{\prime}$ conc $A R$

(12) fi

(13) od

(14) output $\left(w_{j}, w_{j-1}\right)$ conc $T ; \quad$ (* the cyclic adjacency list of vertex $w_{j} *$ )

(15) let $A L=A L^{\prime}$ conc $T^{\prime}$ and $A R=T^{\prime \prime}$ conc $A R^{\prime}$

where $T^{\prime}$ and $T^{\prime \prime}$ contain all edges incident to $w_{j-1}$;

(16) $\quad A L \leftarrow A L^{\prime} ; A R \leftarrow A R^{\prime} ; T \leftarrow T^{\prime} \operatorname{conc}\left(w_{j-1}, w_{j}\right)$ conc $T^{\prime \prime}$

(17) od

(18) $A \leftarrow A R$ conc $\left(w_{0}, w_{k}\right)$ conc $A L$;

(19) return $T$ and $A$

(20) end

Table 1: The procedure embedding

computes a reversed canonical embedding of $S\left(e_{0}\right)$. The call embedding $((1,2), L)$ embeds the entire graph.

The embedding of $S\left(e_{0}\right)$ computed by embedding $\left(e_{0}, t\right)$ is represented in the following nonstandard way:

1. For the vertices $v \in V\left(e_{0}\right)$ we use the standard representation, i.e., the cyclic list of the incident edges corresponding to the clockwise ordering of the edges in the embedding.

2. For the vertices in the stem we use a non-standard representation. For each vertex $w_{i} \in\left\{w_{0}, \ldots, w_{r}\right\}$ let the lists $A L\left(w_{i}\right)$ and $A R\left(w_{i}\right)$ be such that the catenation of $\left(w_{i}, w_{i+1}\right), A R\left(w_{i}\right),\left(w_{i}, w_{i-1}\right)$, and $A L\left(w_{i}\right)$ corresponds to the clockwise ordering of the edges incident to $w_{i}$ in the embedding. Here, $w_{-1}=w_{k}$. Note that $A R\left(w_{i}\right)=\emptyset$ for $1 \leq i<r$ if $t=L$, and $A L\left(w_{i}\right)=\emptyset$ for $1 \leq i<r$, if $t=R$. The lists $A L\left(w_{i}\right)$, $A R\left(w_{i}\right), 0 \leq i \leq r$, are returned in an implicit way: $A L\left(w_{r}\right)$ and $A R\left(w_{r}\right)$ are returned as the list $\bar{T}=A L\left(w_{r}\right),\left(w_{r}, w_{r+1}\right), A R\left(w_{r}\right)$ and the other lists are returned as the list $A=A R\left(w_{r-1}\right), \ldots, A R\left(w_{0}\right),\left(w_{0}, w_{k}\right), A L\left(w_{0}\right), \ldots, A L\left(w_{r-1}\right)$, cf. Figure 3 . 


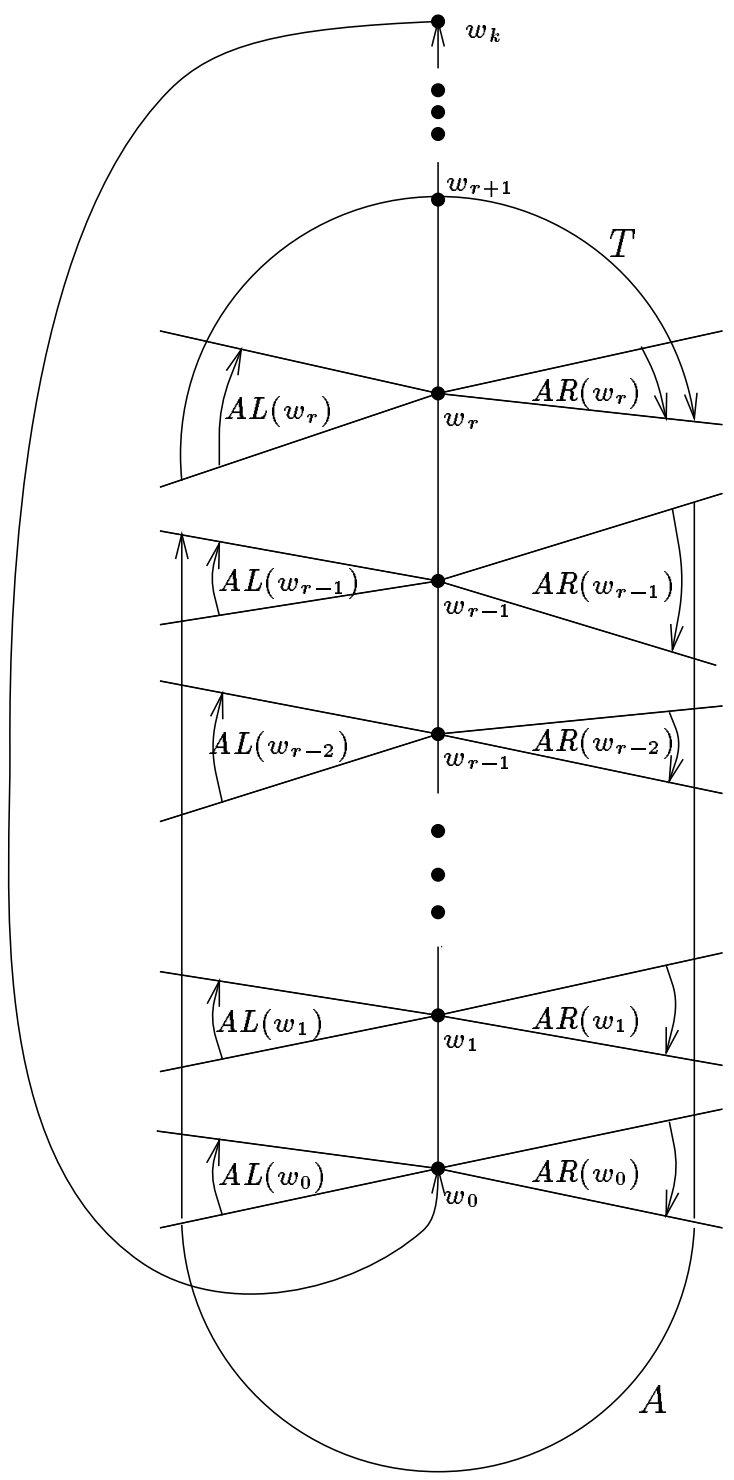

Figure 3: A call embedding $\left(e_{0}, t\right)$ returns lists $T$ and $A$. Lists are drawn as arrows. The arrowhead corresponds to the end of the list. 
The procedure embedding has the same structure as the procedure stronglyplanar and is given in Table 1. It first constructs the stem and the spine (line (1)) of cycle $C\left(e_{0}\right)$, then walks down the spine (lines (3) to (17)), and finally computes the lists $T$ and $A$ to be returned (lines (18) and (19)).

We first discuss the walk down the spine. Suppose that the walk has reached vertex $w_{j}$. We first recursively process the edges emanating from $w_{j}$ (lines (4) to (13)), and then compute the cyclic adjacency list of vertex $w_{j}$ and prepare for the next iteration (lines (14) to (16)).

We discuss lines (4) to (13) first. In general, some number of edges emanating from $w_{j}$ and all edges incident to vertices $w_{l}$ with $l>j$ will have been processed already. Call the processed edges $e_{1}, \ldots, e_{i-1}$. We can now state the invariant of the loop (4) to (13):

- $T$ concatenated with $\left(w_{j}, w_{j-1}\right)$ is the cyclic adjacency list of vertex $w_{j}$ in the embedding of $C+S\left(e_{1}\right)+\ldots+S\left(e_{i-1}\right)$.

- $A L$ and $A R$ are the catenation of the lists $A L\left(w_{0}\right), \ldots, A L\left(w_{j-1}\right)$ and $A R\left(w_{j-1}\right), \ldots$, $A R\left(w_{0}\right)$ respectively where $\left(w_{l}, w_{l+1}\right), A R\left(w_{l}\right),\left(w_{l}, w_{l-1}\right), A L\left(w_{l}\right)$ is the cyclic adjacency list of vertex $w_{l}, 0 \leq l \leq j-1$, in the embedding of $C+S\left(e_{0}\right)+\ldots+S\left(e_{i-1}\right)$.

When $i=0$, i.e., before processing any of the emanating segments the adjacency list of $w_{j}$, $0 \leq j \leq k-1$, is $\left(w_{j}, w_{j+1}\right),\left(w_{j}, w_{j-1}\right)$ and hence $A L\left(w_{j}\right)=A R\left(w_{j}\right)=\emptyset$. We conclude that $T, A L$ and $A R$ are initialized correctly in line (2).

Assume now that we process edge $e^{\prime}=e_{i}$ emanating from $w_{j}$. The flag $\alpha\left(e^{\prime}\right)$ indicates what kind of embedding of $S\left(e_{i}\right)$ is needed to build a canonical embedding of $S\left(e_{0}\right)$; the opposite kind of embedding of $S\left(e_{i}\right)$ is needed to build a reversed canonical embedding of $S\left(e_{0}\right)$. So the required kind is given by $t \oplus \alpha\left(e^{\prime}\right)$, where $L \oplus L=R \oplus R=L$ and $L \oplus R=R \oplus L=R$.

If $e^{\prime}$ is a tree edge, the call embedding $\left(e^{\prime}, t \oplus \alpha\left(e^{\prime}\right)\right)$ computes the cyclic adjacency lists of the vertices in $V\left(e^{\prime}\right)$ and returns lists $T^{\prime}$ and $A^{\prime}$ as defined above. If $e^{\prime}$ is a back edge then $T^{\prime}$ is simply $e^{\prime}$ and $A^{\prime}$ is simply the reversal of $e^{\prime}$. If $S\left(e_{i}\right)$ has to be glued to the left side of the vertical path $w_{0}, \ldots, w_{k}$, i.e., if $t=\alpha\left(e^{\prime}\right)$ then we append $T^{\prime}$ to the front of $T$ and $A^{\prime}$ to the end of $A L$, cf. Figure 4. Analogously, if $S\left(e_{i}\right)$ has to be glued to the right side of the path $w_{0}, \ldots, w_{k}$, i.e., if $t \neq \alpha\left(e^{\prime}\right)$, then we append $T^{\prime}$ to the end of $T$ and $A^{\prime}$ to the front of $A R$. This clearly implements the glueing process described above and also clearly maintains the invariants.

Suppose now that we have processed all edges emanating from $w_{j}$. Then $\left(w_{j}, w_{j-1}\right)$ concatenated with $T$ is the cyclic adjacency list of vertex $w_{j}$ (line (14)).

We next prepare for the treatment of vertex $w_{j-1}$. Let $T^{\prime}$ and $T^{\prime \prime}$ be the list of edges incident to $w_{j-1}$ from the left and from the right respectively and having their other endpoint in an already embedded segment. List $T^{\prime}$ is a suffix of $A L$ and list $T^{\prime \prime}$ is a prefix of $A R$. The catenation of $T^{\prime},\left(w_{j-1}, w_{j}\right), T^{\prime \prime}$, and $\left(w_{j-1}, w_{j-2}\right)$ is the current clockwise adjacency list of vertex $w_{j-1}$. Thus lines (15) and (16) correctly initialize $A L, A R$, and $T$ for the next iteration.

Suppose now that all edges emanating from the spine of $C\left(e_{0}\right)$ have been processed, i.e., control reaches line (18). At this point, list $T$ is the ordered list of edges incident to $w_{r}$ (except $\left(w_{r}, w_{r-1}\right)$ ) and the two lists $A L$ and $A R$ are the ordered list of edges incident to the two sides of the stem of $C\left(e_{0}\right)$. Thus $T$ and the catenation of $A R,\left(w_{0}, w_{k}\right)$, and $A L$ are the two components of the output of embedding $\left(e_{0}, t\right)$. We summarize in

Theorem 1 Let $G=(V, E)$ be a planar graph. Then $G$ can be turned into a planar combinatorial embedding in linear time. 

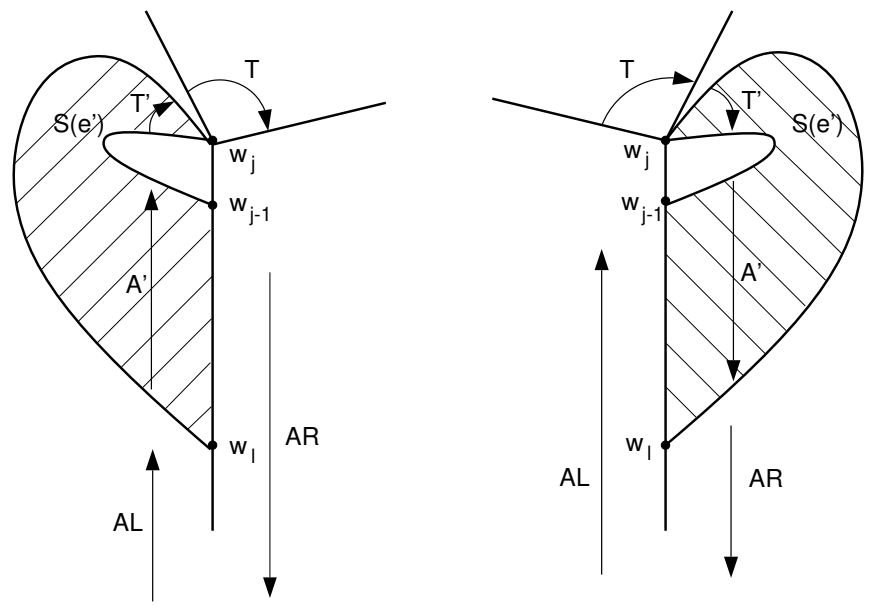

Figure 4: Glueing $S\left(e^{\prime}\right)$ to the left or right side of the path $w_{0}, \ldots, w_{k}$ respectively.

Proof. The correctness follows immediately from the correctness of procedure stronglyplanar, from the fact that stronglyplanar correctly computes the mapping $\alpha$, and from the observation that embedding realizes the glueing process described above.

For the running time analysis we only have to observe that every edge is moved at most once from one of the lists $A L$ and $A R$ onto the list $T$ (onto lists $T^{\prime}$ and $T^{\prime \prime}$ in line (15) and then to $T$ in line (16)), that every edge belongs to at most one spine (line (14)) and that all lines except lines (1) and (15) take constant time.

For an example let us consider the DFS-tree of $G$ given in Figure 1. Consider the situation in the call of embedding $((3,4), L)$. The call embedding $((4,6), L)$ computes the cyclic adjacency lists of the vertices in $V((4,6))$ and returns lists $T^{\prime}=(4,6)$ and $A^{\prime}=(1,6)(2,6)$. In line $(10), T=(4,6)(4,5)$ and $A L=(1,6)(2,6)$. The call of embedding $((4,7), R)$ gives $T^{\prime}=(4,7)$ and $A^{\prime}=(3,7),(1,7)$. Thus in line $(10)$ we have $T=(4,6)(4,5)(4,7)$ and $A R=(3,7)(1,7)$. The adjacency list of node $w_{j}=4$ is completed in line $(14)$. It is $(4,3),(4,6),(4,5),(4,7)$. In line (16) we get $A L=(1,6)(2,6), A R=(1,7)$ and $T=(3,4)(3,7)$. At the end of embedding $((3,4), L)$ we have $A=(1,7)(1,5)(1,6)(2,6)$.

An implementation based on this note is described in [MMN93] and is distributed with the LEDA platform of combinatorial and geometric computing [Näh95, MN95] (anonymous ftp (mpi-sb.mpg.de, directory pub/LEDA)). It first determines the connected and biconnected components and then adds edges to make the graph biconnected. It then tests planarity (using procedure stronglyplanar). If the graph is found to be non-planar, a subdivision of $K_{5}$ or $K_{3,3}$ is identified to prove non-planarity (a trivial method is used for that purpose: the following test is applied to every edge. The edge is removed provisionaly and planarity is tested again. If the graph is still non-planar then the edge is removed. If the graph is now planar the edge is kept. In this way, a subdivision of $K_{5}$ or $K_{3,3}$ is found in quadratic time. We should mention that there is a linear time algorithm to identify a $K_{5}$ or $K_{3,3}$ in a non-planar graph [Wil84]). If the graph is found to be planar a planar combinatorial embedding is constructed. The resulting planar combinatorial embedding is triangulated, a straight-line embedding is constructed (using either the algorithm in [Sch90] or [dFPP91]), and the result is displayed.

The implementation was extensively tested on three kinds of graphs: hand-crafted examples 


\begin{tabular}{|l|r|r|r|r|r|r|}
\hline number of nodes & 1000 & 2000 & 4000 & 8000 & 16000 & 32000 \\
number of edges & 1921 & 3890 & 7842 & 15777 & 31684 & 63558 \\
\hline initializing & 0.33 & 0.80 & 1.88 & 5.02 & 10.88 & 33.02 \\
planarity testing & 0.13 & 0.27 & 0.58 & 1.28 & 2.73 & 6.07 \\
embedding & 0.15 & 0.30 & 0.77 & 1.80 & 4.22 & 9.57 \\
\hline total time & 0.61 & 1.37 & 3.23 & 8.10 & 17.83 & 48.66 \\
\hline
\end{tabular}

Table 2: Running times in seconds on a SUN SPARC 10 for pseudo-random planar graphs. The first row shows the time to prepare the input graph, i.e., to copy it, to make it biconnected and bidirected, the second row shows the time to test planarity, and the third row gives the time for constructing the embedding.

of small size, random sparse graphs, and pseudeo-random planar graphs. The latter graphs were generated by choosing an appropriate number of random line segments in the unit square, computing their intersections, and putting a vertex on every endpoint and intersection. The running time of the implementation is about 50 times the running time of the LEDA strongly connected components algorithm. Table 2 gives more details.

The measured running times grow slightly more than linear. This is due to the increased number of cache faults for larger input graphs. Many of the actions of the algorithm follow the following pattern: an edge, say $(v, w)$, is explored and then a number of labels of node $w$ are inspected and processed. We have chosen LEDA's node arrays to realize node labels. Thus inspecting $k$ node label corresponds to accesses in $k$ arrays and hence to up to $k$ cache faults. Since processing a node label is typically a simple operation these cache faults show up in the measured running time. An alternative implementation where all node labels are stored directly in the node would incur less slow-down due to cache faults.

\section{References}

[BL76] K. Booth and L. Lueker. Testing for the consecutive ones property, interval graphs and graph planarity using $\mathrm{PQ}$-tree algorithms. J. of Computer and System Sciences, 13:335-379, 1976.

[CNAO85] N. Chiba, T. Nishizeki, S. Abe, and T. Ozawa. A linear algorithm for embedding planar graphs using PQ-trees. J. of Computer and System Sciences, 30(1):54-76, 1985.

[dFPP91] H. de Fraysseix, J. Pach, and R. Pollack. How to draw a planar graph on a grid. Combinatorica, 10:41-51, 1991.

[ET76] S. Even and R.E. Tarjan. Computing an st-numbering. Theoretical Computer Science, 2:339-344, 1976.

[FR82] H.de Fraysseix and P. Rosenstiehl. A depth-first-search characterization of planarity. Annals of Discrete Mathematics, 13:75-80, 1982.

[HT74] J. Hopcroft and R. Tarjan. Efficient planarity testing. J. ACM, 21(4):549-568, 1974.

[LEC67] A. Lempel, S. Even, and I. Cederbaum. An algorithm for planarity testing of graphs. Theory of Graphs, Int. Symp.(Rome 1966), pages 215-232, 1967. 
[Meh84] K. Mehlhorn. Data Structures and Efficient Algorithms, volume I, II, III. Springer Verlag, Berlin, 1984.

[MMN93] K. Mehlhorn, P. Mutzel, and St. Näher. An implementation of the Hopcroft and Tarjan planarity test and embedding algorithm. Technical Report MPI-I-93151, Max-Planck-Institut für Informatik, Saarbrücken, 1993.

[MN95] K. Mehlhorn and St. Näher. LEDA: A library of efficient data types and algorithms. CACM, 38(1):96-102, 1995.

[Mut92] P. Mutzel. A fast linear time embedding algorithm based on the Hopcroft-Tarjan planarity test. Technical report, Universität zu Köln, 1992.

[Näh95] St. Näher. LEDA Manual Version 3.1. Technical Report MPI-I-95-1-002, MaxPlanck-Institut für Informatik, 1995.

[Sch90] W. Schnyder. Embedding planar graphs on the grid. In Proc. 1st ACM-SIAM Symp. Discr. Alg. (SODA), San Francisco, pages 138-148, 1990.

[Wil84] S.G. Williamson. Depth-first search and Kuratowksi subgraphs. Journal of the ACM, 11:681-693, 1984. 